\title{
Pour une approche bioculturelle des récits ${ }^{1}$
}

\author{
Marc Lapprand \\ University of Victoria
}

La publication de L'Origine des espèces (1859) a suscité toutes sortes d'interprétations et de tendances dans l'histoire des idées: depuis l'adhésion enthousiaste aux découvertes sur l'évolution jusqu'à l'eugénisme de sinistre mémoire, en passant par un rejet systématique déjà de la part de certains des contemporains de Darwin. Même aujourd'hui, d'aucuns n'y voient dans ses applications au littéraire qu'une sorte de critique historique non déclarée plutôt qu'une réelle approche scientifique en soi; une attitude d'indifférence prédomine encore chez les théoriciens de la littérature (Saul, p. 112 et

1 Je tiens à remercier mes collègues Kim Blank, Alain Schaffner, Insook Webber et Martin Winckler pour leurs précieux conseils. 
125). Il est remarquable, pourtant, que très tôt des critiques littéraires aient modelé leurs travaux sur la théorie de l'évolution. Hippolyte Taine (1828-1893) est l'un des premiers à avoir été directement influencé par Darwin. Il proposait une forme de critique littéraire physiologique prenant en compte la race (au sens d'«empreinte d'un esprit national»), le milieu (environnements physique et historique) et le moment : " Il en résulte une psychologie scientifique, que l'on peut étudier en botaniste ou en anatomiste, et qui vise à retrouver chez l'écrivain le secret de son mécanisme mental. » (Bergez, p. 15) À la même époque, et dans la mouvance du naturalisme, c'est Zola qui appuie massivement son histoire des Rougon-Macquart sur l'hérédité et donc, dans une certaine mesure, sur l'évolution.

Cependant, le «darwinisme » tel que les intellectuels non strictement scientifiques le percevaient à la fin du dix-neuvième siècle a produit une espèce de cacophonie où se mêlaient dérives idéologiques et sciences naturelles. Même le concept de «lutte pour la survie » en est venu à être parfois considéré au niveau de l'individu, au lieu de l'espèce, ce qui n'a guère de sens. Daniel Grojnowski le note dans sa préface à L'Enquête sur l'évolution littéraire de Jules Huret (1891) : «Raisonnant, peutêtre sans trop s'en rendre compte, en adepte du darwinisme, J. Huret est amené à concevoir le champ culturel comme un espace conflictuel qui voit triompher les mieux adaptés, les plus forts ou les plus féroces.» (p. 20) Fortement inspiré par Brunetière, lui-même adepte du transformisme de Lamarck, Huret, plus proche de Sainte-Beuve que de Darwin, menait ses enquêtes sans souci "évolutionniste» autre que ce que lui suggérait une mode rendue attrayante par l'emploi d'un jargon pseudo-scientifique. Au début du vingtième siècle, certains intellectuels de premier plan ont dès lors pris une position 
nuancée par rapport à l'évolution. C'est le cas de Valéry, qui, pourtant, reconnaissait le bouleversement potentiel que la théorie de Darwin était à même d'apporter dans notre conception du monde ${ }^{2}$. C'est aussi celui de Bergson, qui, à la même époque dans L'Évolution créatrice (1907), examinait les tendances du néodarwinisme tout en distinguant l'intellect humain du biologique.

Les dérives idéologiques auxquelles le darwinisme fut associé dans l'entre-deux-guerres mirent peut-être un frein aux explorations de cette discipline dans les sciences humaines et sociales. Cette théorie ne figurait en tout cas certes pas au programme de la Nouvelle Critique américaine ou du formalisme de l'École de Prague. Ce n'est véritablement que depuis le début des années 1990 - surtout dans le monde anglo-saxon - qu'est en train de se former une nouvelle discipline qui propose un autre mode de lecture des textes littéraires, appelé parfois «biopoétique» ou «darwinisme littéraire », depuis l'ouvrage clé de Joseph Carroll du même titre, en rapport direct avec les derniers développements de la psychologie évolutionniste. Cette nouvelle approche offre en corollaire une remise en cause de certaines méthodes critiques qui ont dominé le discours académique pendant deux générations successives, du structuralisme à la déconstruction. Les découvertes récentes en neuroscience et en psychologie cognitive ne sont pas étrangères à ce nouveau créneau

\footnotetext{
2 «Ceci nous ramène à Darwin sans détour. Celui-là je ne l'aime pas, il m’est indifférent et comme extérieur. Mais nul historien ne peut ignorer son existence. Songe que s'il a raison, toute l'histoire en est changée. Je veux dire tout le raisonnement historique. Et il est certain qu'il a apporté quelque chose. » (Valéry, Lettre à son ami André Lebey [1906], dans 1960, p. 15441545)
} 
scientifique, car jamais n'avons-nous été mieux informés sur le fonctionnement réel du cerveau humain (Saul, p. 300). On l'aura compris, ce nouveau mode de réflexion veut intégrer le biologique avec le littéraire, le génétique avec l'ontologique. En effet, il faut partir du biologique avant de considérer le social, car l'être humain, tout en étant hautement socialisé, est d'abord un animal dont certains comportements sont encore fortement engrammés dans son génome.

Vers la fin du vingtième siècle, un certain radicalisme théorique a abouti à une forme de déterminisme culturel qui a fait de l'individu un produit de sa culture, comme s'il partait de zéro à la naissance. Or si la culture du milieu dans lequel nous sommes nés façonne plus ou moins notre mode de vie en société (facteurs épigénétiques), elle ne saurait supplanter un bagage génétique qui nous est propre, non en tant qu'individu, mais en tant qu'espèce. L'évolutionnisme redonne la primauté à tout ce qu'il y a de biologique en nous, notamment d'un point de vue phénotypique, ce terme désignant les «traits individuels résultant de l'interaction entre l'environnement d'un organisme et son génotype» (Workman, p. 335). Dans ce sens, nous assistons bel et bien à un mouvement radicalement contraire au fonctionnement très compartimenté dans lequel opéraient les divers savoirs en sciences humaines et sociales pendant la seconde moitié du siècle dernier et où les excès de spécialisation ont produit des aberrations notoires dans le métadiscours littéraire, les mathématiques étant ainsi parfois invoquées en vue d'obscurcir le métadiscours. Cela a produit des impasses, certes, mais celles-ci ont aussi eu pour effet positif d'hybrider les discours, en suscitant entre autres le retour de la critique historique et l'avènement de la critique génétique, sous 
l'impulsion des travaux fondateurs de Pierre-Marc de Biasi sur les manuscrits de Flaubert.

L'un des fondements de cette approche renouvelée réside dans la « consilience ». Le terme n'est pas nouveau; il fut créé par un philosophe scientifique anglais, William Whewell, en 1840 (Gould, p. 192). Or c'est le célèbre entomologiste et père de la sociobiologie, Edward O. Wilson, qui va redonner un nouveau lustre à ce terme, en l'utilisant comme titre d'un de ses ouvrages (1998; en français "l'unicité du savoir »). Pour Wilson, qui en modifie légèrement l'acception originale, la consilience désigne l'imbrication des explications causales dans le but de réunir et d'harmoniser les champs de la connaissance (sciences pures, humaines et sociales), au lieu de les séparer et de les radicaliser selon les tendances précédentes. Pour lui, il s'agit du défi ultime de la pensée humaine: faire se rejoindre les sciences et les humanités dans un "même élan » (selon l'étymologie du terme « consilience ») de pensée synthétique, la sociobiologie en étant la discipline matricielle par excellence. Deux précurseurs de la littérature d'anticipation illustrent d'ailleurs à merveille une forme de consilience : H.G. Wells et Jules Verne.

Cette notion de consilience est globalisante et synthétisante: elle aspire à ce que toutes les sciences convergent pour lire et dire notre condition humaine, comprendre qui nous sommes et où nous allons. La lecture de notre génome nous permettra peut-être de tout rassembler, au lieu de continuellement diviser les savoirs dans des zones hermétiques. Nos différences culturelles, raciales, sociales ne sont que des écrans de fumée : nous sommes tous des cousins éloignés de Lucy, la célèbre australopithèque dont on a retrouvé les restes en 1974 en Éthiopie. Mieux encore, selon Steve Olson, 
la totalité de la population terrestre actuelle descendrait de quelque 20000 individus qui peuplaient la corne de l'Afrique, il y a environ 200000 années (Olson, p.28). Quelle que soit la polémique que ce chiffre a pu soulever parmi les anthropologues, la plupart s'accordent à avancer que, selon le lignage mitochondrial présent dans toute séquence d'ADN humaine, nous descendons tous effectivement des mêmes ancêtres. Certes, selon les régions du globe, au gré des migrations humaines et des métissages ethniques, les peuples ont évolué en affichant des traits parfois distinctifs et reconnaissables. Nous ne formons pourtant qu'une seule espèce, ce qui devrait suffire à (idéalement) anéantir le terme de "race», qui n'a plus aucun fondement scientifique. Claude Lévi-Strauss l'avait déjà pressenti en son temps, en invitant instamment à ne pas mélanger race et culture :

Aussi, quand nous parlons [...] de contribution des races humaines à la civilisation, ne voulons-nous pas dire que les apports culturels de l'Asie ou de l'Europe, de l'Afrique ou de l'Amérique tirent une quelconque originalité du fait que ces continents sont, en gros, peuplés par des habitants de souches raciales différentes. Si cette originalité existe - et la chose n'est pas douteuse - elle tient à des circonstances géographiques, historiques et sociologiques, non à des aptitudes distinctes liées à la constitution anatomique ou physiologique des noirs, des jaunes ou des blancs (Lévi-Strauss, p. 10-11).

\section{Vers une approche évolutionniste de la littérature}

Ce n'est pas seulement le texte littéraire dans sa forme canonique qui est concerné : il s'agit de rendre compte du phénomène universel qu'est le fait de raconter des histoires. Un ouvrage récent de Brian Boyd, spécialiste reconnu de Nabokov, 
a démontré avec autant de brio que de conviction ce que peut produire la consilience en études littéraires : On the Origin of Stories (2009). Le fait que ce livre soit sorti au centcinquantième anniversaire de L'Origine des espèces n'est pas le fruit du hasard. S'appuyant sur des principes sociobiologiques et évolutionnistes dans son étude du littéraire, Boyd explore de nouveaux modes de lecture, en délaissant un tant soit peu l'analyse purement sémiologique ${ }^{3}$. Il propose, faute de mieux pour le moment, le mot «evocriticism» (Boyd, p. 384) pour désigner cette nouvelle science de la littérature. Après une première partie théorique, Boyd étudie en guise d'application deux textes que rien ne rapproche d'emblée: L'Odyssée d'Homère et Horton Hears A Who! du fameux Dr. Seuss (1954). Dans le premier cas, la question fondamentale qu'il se pose est de comprendre ce qui justifie le pouvoir d'attraction jamais démenti de cette narration trimillénaire. Pourquoi l'être humain s'intéresse-t-il encore à l'histoire du retour d'Ulysse chez lui après vingt ans de pérégrinations qui ne peuvent en rien correspondre à notre mode de vie contemporain (valeurs individuelles, morales, guerrières, philosophiques)? Pour le second, Boyd propose que l'album illustré pour enfants de Seuss est une métaphorisation de l'humiliation cinglante vécue par les Japonais après leur défaite à l'issue de la Seconde Guerre mondiale (Hiroshima, Nagasaki). La première est une étude phylogénique (origine évolutive) et la seconde ontogénique (origine individuelle), ce qui justifie leur rapprochement pour mieux les contraster.

\footnotetext{
3 Certains détracteurs du darwinisme littéraire arguent de son silence sur le plan esthétique, car sont mis au même niveau le canon et la littérature populaire (voir Adams, p.155-170). N'est-ce pas ce que l'on reprochait naguère aux structuralistes?
} 
Trois grands principes gouvernent la méthode : (a) l'art est une "machine darwinienne» (Boyd, p.121) qui existe pour des raisons biologiques; (b) la fiction correspond à une expérience cognitive personnalisée; (c) les récits, dans toute leur variété, sont autant d'atouts majeurs pour les prises de décision, le fictif servant à informer le réel et aidant à agir sur celui-ci. Dans une perspective évolutionniste, l'art et, en particulier, la littérature deviennent donc un facteur performant pour faciliter l'adaptabilité de l'être humain, notamment sa capacité de raisonner (Gottschall et Wilson, p. 169). Pour les évolutionnistes, le cerveau, dans toute sa complexité, est « adapté ». Pour dire les choses autrement, si l'être humain est capable d'une activité mentale d'une complexité qu'aucun animal ne peut égaler, c'est le fruit d'une adaptation, même si celle-ci est sans doute due à un hasard évolutif. Mais pourquoi notre cerveau a-t-il ainsi évolué4 ?

Poussons d'un cran ce questionnement: pourquoi sommes-nous plus souvent séduits ou attirés par les fictions, les récits imaginaires, fantasmés, utopiques ou même surnaturels (et religieux), que par les récits écrits ou filmiques documentaires, scientifiques ou purement informatifs? En corollaire, pourquoi avons-nous inventé les dieux et les religions (voir Boyer, 2001) ? Tout lecteur est un analyste en puissance, même si ses outils sont intuitifs avant d'être formalisés. Les évolutionnistes s'attachent à décrire les processus cognitifs en jeu lors de la lecture et, en cela, se trouvent grandement assistés par les neurobiologistes qui, en

\footnotetext{
4 Question qui a justifié l'imposante étude de l'anthropologue évolutionniste Terrence Deacon (1997), qui propose notamment que notre cerveau a progressivement adapté (en l'élargissant) la zone préfrontale du cortex pour satisfaire les besoin neurocognitifs liés à l'acquisition et la maîtrise du langage.
} 
parallèle, montrent le cerveau en train de réagir à des stimuli spécifiques. On est ainsi capable, grâce à l'imagerie par résonance magnétique (IRM) et la tomographie par émission de positrons (TEP), de cartographier l'activité corticale et de mettre en évidence l'intérêt vital que l'être humain porte aux valeurs que sont le jeu, l'art, la créativité, et l'activité cérébrale purement imaginaire, dans des optiques de compétition, de coopération, de désir de reconnaissance et de statut social. Ce sont tous ces aspects que veut révéler l'approche bioculturelle des récits, afin de démontrer les fonctions fondamentalement humaines de ces comportements, qui en l'occurrence transcendent, sans pour autant le nier, le «culturel» que chacun de nous a intériorisé. Les fictions que nous assimilons dès notre plus jeune âge activent en nous certaines fonctions vitales, en préparant nos circuits neurocognitifs à faire face à des situations pour lesquelles nous sommes déjà programmés (sélections naturelle et sexuelle, par exemple), mais aussi des situations auxquelles nous n'avons jamais été confrontés (peurs du vide et de l'étranger, répulsion face aux serpents ou aux araignées, par exemple) et qu'il faut vivre une première fois afin d'en activer le gène porteur. C'est ce que les évolutionnistes nomment la «théorie de l'alerte préventive» (Workman, p. 263).

$\mathrm{Si}$, faute de traces paléoanthropologiques suffisamment abondantes, il est extrêmement ardu de dater les moments clés dans les changements majeurs de notre évolution, il a cependant été possible d'établir que les pratiques ludiques et artistiques précèdent de très loin l'avènement du langage, qui est le propre de notre espèce. Les anthropologues ont montré que les récits ont pu exister sans autre forme de communication 
que la danse, le mime et l'expression gestuelle, voire les tatouages ou les peintures corporelles: on a ainsi trouvé des traces d'utilisation de l'ocre sur la peau remontant à près de 120000 ans (Boyd, p. 77), tandis que les peintures murales de la grotte de Chauvet (France) datent de quelque 30000 ans. Il va sans dire que l'apparition et le développement du langage ont progressivement démultiplié les possibilités narratives.

L'anthropologue et psychologue britannique Robin Dunbar a eu l'idée originale, sinon lumineuse, de lier la naissance du langage à la pratique de l'épouillage entre nos ancêtres les grands singes. Cette activité en effet ne peut se pratiquer que par couple. Au-delà de sa raison d'être purement hygiénique, on sait que c'est aussi un mode de communication très efficace entre deux individus et qui est proche à ses débuts, selon Dunbar, d'une forme de commérage («gossip »). En outre, «l'épouillage devient une mesure fiable de dévouement et peut être payé de retour par une assistance lors d'agressions » (Nadel, p. 14). Or il a bien fallu trouver un autre medium pour s'adresser en même temps à plusieurs individus, d'où l'apparition du langage, qui a sans doute dû débuter par des grognements, raclements de gorges et cris articulés (Dunbar, Grooming, Gossip, 1997). Si l'on est encore incapable de situer dans le temps l'apparition de la grammaire des langues, même approximativement, on est néanmoins certain qu'elle devance largement l'arrivée de l'écriture.

Les évolutionnistes s'interrogent sur la raison d'être de ce qui fait de notre espèce le seul animal parlant, avec pour conséquence immédiate notre propension à entendre ou à lire des histoires et, partant, à en raconter. Même si la linguistique saussurienne a récemment modifié notre perception du langage 
en l'envisageant essentiellement comme un moyen de communication, les anthropologues et les sociologues ont établi que la grande majorité de nos prises de parole quotidiennes sont de l'ordre du babillage ou du commérage, ce qui ressortit à la fonction phatique déjà identifiée par Jakobson dans les années 1960, bref d'informations a priori non pragmatiques et n'ayant que peu à voir avec nos besoins réels comme la nourriture ou la sécurité. La perception de ce type de discours change cependant si l'on considère qu'il s'agit avant tout de raconter des histoires, vraies ou controuvées.

C'est à partir de l'âge de quatre ou cinq ans que l'enfant est normalement capable d'inférer correctement ce que l'autre pense et, en particulier, de reconnaître avec assurance si l'histoire racontée est véridique ou inventée. C'est là une application directe de la «psychologie intuitive» («theory of mind», Workman, p.115), qui fait malheureusement défaut chez les enfants autistes. Dès ce moment se développe chez l'être humain une appétence incontrôlable pour les histoires racontées et pour, en particulier, celles du type «faire-commesi », ce que Jean-Marie Schaeffer (p. 41 et suiv.) appelle la feintise ludique. Comment la narration peut-elle être considérée comme un phénomène d'adaptation à l'environnement et non un simple sous-produit de l'aptitude au langage?

Darwin fut le premier à proposer que l'art, sous n'importe quelle forme, en raison particulièrement de son coût élevé, voire de son extravagance, est un facteur caché jouant en faveur de la sélection sexuelle (Miller, 2001, p. 260-261). En effet, si un individu est capable de consacrer autant d'énergie à une chose reconnue comme étant inutile pour la survie quotidienne, ce ne 
peut être que la preuve qu'il a d'excellents gènes : il peut se permettre de gaspiller ses efforts là où d'autres renâclent à des tâches purement utilitaires (abri, nourriture, soins basiques). Il s'avère donc un partenaire éminemment attrayant en période génitale, c'est-à-dire à l'âge optimal pour la reproduction. Nous possédons des traces d'artefacts artistiques remontant à l'âge de pierre (hachettes ornementées, statuettes humaines ou animales) dont on sait qu'ils n'avaient aucune visée utilitaire, en raison de leur abondance dans un même emplacement. On en est venu à supposer que ces manifestations artistiques étaient loin d'être aussi gratuites, ou uniquement esthétiques, qu'elles pouvaient le paraître d'emblée. Nous avons pourtant affaire ici à un contentieux entre les évolutionnistes. Le psychologue évolutionniste Geoffrey Miller, par exemple, penche pour la théorie darwinienne de l'art comme stratégie de sélection sexuelle. Wilson, quant à lui, considère l'art (et la religion) comme le produit d'un cerveau sur-adapté en quelque sorte. Tout se passe alors comme si le mental devait créer de l'art pour compenser sa complexité innée (Carroll, p. xx-xxi).

Si l'on accepte l'hypothèse que l'art en général est une adaptation, favorisant la survie et la reproduction du plus apte, principes darwiniens par excellence, qu'en est-il plus spécifiquement de la narration et donc de la pratique littéraire?

En inventant (ou en écrivant) des fictions, nous voulons avant tout partager avec d'autres auditeurs ou lecteurs potentiels les histoires dans lesquelles sont médiatisées des sélections et des combinaisons de toute la panoplie des sentiments et des émotions. Au-delà des stratégies narratives conscientes chez l'auteur de la fiction existe aussi le désir de partage et de coopération, que renforce le statut social conféré 
par les lecteurs aux créateurs de fictions, ce qui augmente leur visibilité et satisfait du même coup leur soif de reconnaissance. Selon Geoffrey Miller, les indicateurs de l'aptitude et de la propension à raconter des histoires de manière accomplie ou réussie servent de méta-fonctions mentales par rapport aux adaptations basiques de l'être humain à son environnement naturel (Miller, 2001, p. 105). Ils constituent à cet égard une sorte de plus-value adaptative. Certes, pour satisfaire cette fonction, il nous faut des histoires originales, inventives, d'un imaginaire renouvelé, des récits qui nous interpellent, nous étonnent, nous choquent, nous perturbent, nous effraient ou nous hypnotisent...

Geoffrey Miller insiste sur cette disposition au langage sous toutes ses formes (langage oral ou écrit, romans ou films), car il la considère comme un véritable indicateur adaptatif dans la sélection sexuelle. Il prend comme illustration exemplaire d'étalage verbal («verbal display») les cinq niveaux discursifs de l'histoire de Cyrano de Bergerac: 1) l'auteur original de L'Histoire comique des états et empires de la lune (1657); 2) la pièce d'Edmond Rostand, Cyrano de Bergerac (1897); 3) le personnage de Cyrano dans toute sa verve séductrice destinée à gagner le cœur de Roxane; 4) le traducteur anglais de la pièce (Anthony Burgess,puisque c'est en anglais que Miller l'a lue); 5) Geoffrey Miller (2001, p. 378) lui-même qui relate tous ces niveaux dans son ouvrage The Mating Mind. Cette chaîne peut même se prolonger par l'auteur de ces lignes qui la commente à son tour, puis par le lecteur de ces lignes et éventuellement par d'autres à qui elles seraient transmises. De tels niveaux de discursivité, qui remplissent tous des fonctions adaptatives, 
peuvent aisément s'appliquer à d'autres grandes œuvres du passé, transcendant le dispositif purement textuel mis en place.

Une lecture évolutionniste va donc faire éclater le cadre rigide du texte, valoriser la subjectivité des récepteurs de l'œuvre et poser autant de points de vue (loci) que de niveaux discursifs, comme dans l'exemple de Cyrano, sans privilégier la sémiotique textuelle en soi. Le texte devient l'un des pôles adaptatifs d'un ensemble cohérent dont toutes les composantes sont à considérer (individu, écrivain créateur, narrateur, personnage ou narrataire, histoire racontée, lecteur, critique). Il ne s'agit certes pas de restituer ici des égalités simplistes du type auteur = narrateur, mais de faire intervenir tous ces pôles en harmonisant leurs relations dans la perspective des nécessités adaptatives. La finesse que permet d'atteindre une analyse purement textuelle reste tout à fait valable et nécessaire dans une perspective évolutionniste, car on ne peut jamais épuiser la richesse d'interprétation des histoires auxquelles nous sommes confrontés. Et c'est justement l'un des premiers motifs de cette approche, qui consiste non pas tant à établir des points de vues discursifs (auteur - personnage lecteur) que des interprétations des situations engagées par l'histoire racontée, notamment grâce à la psychologie intuitive (Carroll, p. 165). Ce qui est particulièrement prégnant ici, c'est que cette aptitude humaine à inférer ce que l'autre pense s'applique également aux trois loci indiqués plus haut entre parenthèses: le lecteur suppute ce que pense l'auteur et réciproquement, de même qu'il raisonne sur ce que " pensent » les personnages, autant unilatéralement qu'entre eux. 


\section{Vers une méthodologie bioculturelle?}

Après avoir souligné ces grandes lignes de la psychologie évolutionniste, peut-on parler d'une méthodologie relativement à la lecture d'un texte littéraire ? On aura compris qu'il s'agit avant tout d'une méthode empirique, fondée sur l'observation des faits et qui ne saurait présumer des résultats qu'elle escompte. Les chercheurs, dans ce domaine, s'accordent à penser que l'évolutionnisme en est au seuil de la phase pédagogique, celle de la production de manuels destinés à l'enseignement universitaire (Carroll, p. xix). Cela signifie qu'un effort est actuellement en cours en vue de la diffusion de ces découvertes relativement récentes, mais que cette discipline n'a pas encore atteint une maturité telle qu'elle puisse prétendre à une théorie constituée. On voit d'ailleurs se dégager deux courants divergents: l'un généraliste, représenté notamment par Boyd, Carroll, Gottschall et E.O. Wilson, et l'autre plus restreint, tel que l'envisagent surtout Buss, Cosmides et Tooby (voir Scher et Rauscher). Pourtant, les débats actuellement en cours sont tels qu'il est plausible que cette prochaine phase (théorique) soit déjà bien en voie de se constituer. L'évolutionnisme a remis en cause un certain nombre de théories sur la base de leur contradiction intrinsèque (le postmoderne) ou de leur inanité biologique (le complexe d'CEdipe). Le plus grand paradoxe à résoudre est peut-être celui $\mathrm{du}$ déterminisme culturel, qui présente la liberté humaine comme illusoire. Il ne s'agit pas de nier le culturel comme spécificité acquise, mais de reconnaître qu'il y a d'abord et avant tout une nature humaine et que celle-ci conditionne pour une part nos agissements. Cela n'explique évidemment pas tout : ce n'est pas parce que nous sommes dotés de deux jambes 
pour marcher qu'est prédéterminé l'endroit où nous allons nous rendre dès lors que nous nous mettons à marcher.

Jean-Marie Schaeffer posait lui aussi au tournant du siècle dernier la question de savoir pourquoi l'être humain a une telle soif de fiction, mais son point de vue ne débordait guère le cadre de la littérature. Le biologique y affleurait parfois, mais ce n'est pas une avenue explorée de propos délibéré par l'auteur et qui n'est pas toujours favorable à des concepts tels que celui de «mème » élaboré par Richard Dawkins pour faire pendant au gène, analogie que Schaeffer estime en l'occurrence «trop simpliste» (Schaeffer, p.121). Mais on ne peut ignorer la richesse et la pertinence de son propos, notamment sur le plan de ce qu'il appelle la modélisation des jeux fictionnels, qui concorde avec les enjeux évolutionnistes.

Joseph Carroll propose par exemple une structure de base comportant cinq concepts nécessaires et suffisants pour amorcer une lecture évolutionniste :

(a) la nature humaine en tant que hiérarchie de motifs structurée (au sein de laquelle le motif de construire des représentations imaginaires tient une place prééminente); (b) le "point de vue», ou la topique du sens selon trois centres de conscience distincts : celui de l'auteur, des personnages et du lectorat pressenti; (c) le recours à des universaux humains comme cadre général de référence par rapport auquel les auteurs posent leur propre identité de même que leurs propres structures signifiantes individuelles; (d) un ensemble de catégories destiné à analyser les différences individuelles identitaires; et (e) la distribution d'un sens littéraire spécifique réparti en trois dimensions principales: (i) le thème (contenu conceptuel), (ii) le ton (ambiance émotionnelle) et (iii) l'organisation formelle (une notion qui s'étend des macrostructures telles que l'intrigue et 
l'enchaînement narratif jusqu'aux microstructures comme le phrasé, le lexique et les séquences sonores ${ }^{5}$.)

C'est cette grille de lecture qui m'a permis d'échafauder un essai sur l'œuvre de Martin Winckler. L'ouvrage vise à montrer ce qui suscite chez Marc Zaffran la nécessité de se parer d'un pseudonyme d'écrivain dont le nom est emprunté à l'œuvre de Georges Perec (Winckler), de créer un personnage de médecin fortement inspiré de son expérience personnelle et qui apparaît dans plusieurs romans (Bruno Sachs), le tout afin de légitimer son activité d'écriture et d'espérer atteindre la renommée littéraire. La cohésion de ces trois pôles est telle que, tout en les distinguant sur le plan narratologique, il ne semble guère viable en l'occurrence de les distinguer totalement, sauf à produire des aberrations. Ainsi par exemple, on sait à quel point l'éthique médicale en est un leitmotiv de première importance. Or comment concevoir que le personnage de Bruno Sachs, dont il est le porte-drapeau, n'épouse pas le credo de Marc Zaffran lui-même ? Parce que les romans « médicaux » de Martin Winckler sont réalistes dans leur conception, il est nécessaire que l'écrivain et le personnage central croient aux

5 « (a) human nature as a structured hierarchy of motives (within which the motive of constructing imaginative representations holds a prominent place); (b) "point of view", or the location of meaning within three distinct centers of consciousness - that of the author, the characters, and the implied or projected audience; (c) the use of human universals as a common frame of reference in relation to which authors identify their own individual identities and their own distinct structures of meaning; (d) a set of categories for analyzing individual differences in identity; and (e) the distribution of specifically literary meaning into three chief dimensions: (i) theme (conceptual content), (ii) tone (emotional coloring), and (iii) formal organization (a concept that ranges all the way from macrostructures like plot and narrative sequencing to microstructures like phrasing, word choice, and sequences of sounds). » (Carroll, p. 188, je traduis) 
mêmes valeurs, sauf à tomber dans une incohérence totale ou, pire, dans une parodie macabre dont on sait avec certitude que tel n'est pas l'objet de son auteur (voir Lapprand, 2012).

Nul ne le niera, par-delà nos ascendances familiales, sociales, culturelles et ethniques, chacun de nous a soif d'entendre et de lire des histoires. À ce niveau-là rien n'a vraiment changé depuis L'Iliade et L'Odyssée d'Homère jusqu'à Harry Potter et Millenium. À nous d'en tenir compte aujourd'hui devant tout texte littéraire, d'où qu'il provienne. Les interprétations sont infinies, aussi illimitées que l'imaginaire humain. Notre consommation narrative est un besoin vital, adaptatif et donc profondément évolutif. Heureusement pour nous, Schéhérazade ne s'est pas arrêtée après la mille-etunième nuit. Ses histoires continuent de traverser siècles et frontières, elles ont simplement changé de couleur, de vernis et de conteur. Tant qu'il y a aura de l'humain, il y aura du cognitif et, par conséquent, du narratif. Il s'agit en somme de rien de moins que la survie du notre cerveau, donc de notre espèce. 


\section{Bibliographie}

Cette bibliographie, sans vouloir être exhaustive, répertorie une sélection d'ouvrages majeurs dans le domaine de la psychologie évolutionniste. Le cas échéant, la version française est indiquée. Nous avons placé dans une catégorie à part les ouvrages qui touchent directement au domaine littéraire.

\section{Ouvrages généraux}

ADAMS, Jon. (2011), «Value Judgments and Functional Roles: Carroll's Quarrel with Pinker», dans Nicholas SAUL et Simon J. JAMES (dir.), The Evolution of Literature: Legacies of Darwin in European Cultures, Amsterdam, Rodopi.

BERGEZ, Daniel et al. (2002), Méthodes critiques pour l'analyse littéraire, Paris, Nathan Université.

Blackmore, Susan. (1999), The Meme Machine, Oxford, New York, Oxford University Press. Traduction française: La Théorie des mèmes: Pourquoi nous nous imitons les uns les autres, Paris, Max Milo, 2006.

Boyer, Pascal. (2001), Et l'homme créa les dieux: Comment expliquer la religion, Paris, Gallimard, coll. « Folio essais ».

BRYSON, Bill. (2007), A Short History of Nearly Everything. New York, Broadway Books, 2004. Traduction française: Une histoire de tout, ou presque..., Paris, Payot.

Buican, Denis. (2008), L'Odyssée de l'évolution, Paris, Ellipses.

Buss, David. (1999), Evolutionary Psychology: The New Science of Mind, Boston, Allyn and Bacon. 
DARWIN, Charles. ([1859] 2009), On The Origin of Species by Means of Natural Selection, New York, The Modern Library, présentation d'Edward J. Larson (nombreuses éditions en français de L'Origine des espèces et dans d'autres langues).

DAWKInS, Richard. (1975), The Selfish Gene, Oxford University Press. Traduction française : Le Gène égoïste, Paris, Odile Jacob, 1976.

DEACon, Terrence. (1997), The Symbolic Species, New York, Londres, W. W. Norton and Company.

DUNBAR, Robin. (1997), Grooming, Gossip, and the Evolution of Language, Cambridge (Mass.), Harvard University Press.

-. (2004), The Human Story: A New History of Mankind's Evolution, Londres, Faber and Faber.

- et Louise BARRETT (dir.). (2007), The Oxford Handbook of Evolutionary Psychology, Oxford, Oxford University Press.

FISHER, Helen. (1992), Anatomy of Love, New York, Ballantine Books, traduction française : Histoire naturelle de l'amour, Paris, Robert Laffont, 1994.

Gould, Stephen Jay. (2003), The Hedgehog, the Fox and the Magister's Pox: Mending the Gap between Science and the Humanities, New York, Three Rivers Press.

HuRET, Jules. (1999), Enquête sur l'évolution littéraire, Paris, José Corti.

LÉvi-STRAuSs, Claude. (1961), Race et Histoire, Paris, Éditions Gonthier.

Miller, Geoffrey. (2001), The Mating Mind: How Sexual Choice Shaped the Evolution of Human Nature, New York, Anchor Books.

-. (2009), Spent: Sex, Evolution, and Consumer Behavior, New York, Viking. 
NADEL, Jacqueline et Jean DECETY. (2002), Imiter pour découvrir l'humain: Psychologie, neurobiologie, robotique et philosophie de l'esprit, Paris, PUF.

Olson, Steve. (2002), Mapping Human History: Genes, Race, and Our Common Origins, Boston, New York, Houghton Mifflin Company.

PINKER, Steven. (2002), The Blank Slate: The Modern Denial of Human Nature. New York, Viking Penguin, traduction française: Comprendre la nature humaine, Paris, Odile Jacob, 2005.

Raymond, Michel. (2008), Cro-Magnon toi-même!: Petit guide darwinien de la vie quotidienne, Paris, Seuil, coll. «Points Sciences ».

ScHER, Steven J. et Frederick Rauscher (dir.). (2003), Evolutionary Psychology: Alternative Approaches, Boston, Dordrecht, New York, Londres, Kluwer Academic Publishers.

SmAIL, Daniel Lord. (2008), On Deep History and the Brain, Berkeley, Los Angeles, Londres, University of California Press.

VAlÉRY, Paul. (1960), CEuvres II, Paris, Gallimard, coll. «Bibliothèque de la Pléiade ».

WILSON, Edward O. (1998), Consilience: The Unity of Knowledge, New York, Akfred A. Knopf, traduction française : L'Unicité du savoir: de la biologie à l'art, une même connaissance, Paris, Robert Laffont, 2000.

WORKMAN, Lance et Will Reader. (2004), Evolutionary Psychology: An Introduction, Cambridge, Cambridge University Press, traduction française: Psychologie évolutionniste: Une introduction, Bruxelles, De Boeck, 2007. 


\section{Ouvrages plus spécifiques aux approches évolutionnistes du narratif ou de l'art}

BoyD, Brian. (2009), On the Origin of Stories: Evolution, Cognition and Fiction, Cambridge (Mass.), Londres, Harvard University Press.

CARRoLL, Joseph. (1995), Evolution and Literary Theory, University of Missouri Press.

-. (2004), Literary Darwinism: Evolution, Human Nature, and Literature, New York, Routledge.

DutTon, Denis. (2009), The Art Instinct, New York, Berlin, Londres, Bloomsbury Press.

GotTSChALL, Jonathan. (2003), "The Tree of Knowledge and Darwinian Literary Studies", Philosophy and Literature, vol. 27, no 2, p. 255-268.

- et David Sloan Wilson (dir.). (2005), The Literary Animal: Evolution and the Nature of Narrative, Evanston (Illinois), Northwestern University Press.

LAPPRAND, Marc. (2012), Trois pour un: Une lecture évolutionniste de l'œuvre de Martin Winckler, Québec, Presses de l'Université du Québec.

SAUL, Nicholas et Simon J. James (dir.). (2011) The Evolution of Literature: Legacies of Darwin in European Cultures, Amsterdam, New York, Rodopi.

SCHAEFFER, Jean-Marie. (1999), Pourquoi la fiction?, Paris, Seuil.

ToobY, John et Leda Cosmides. (2001), “Does Beauty Build Adapted Minds? Toward an Evolutionary Theory of Aesthetics, Fiction and the Arts", SubStance, vol. 30, no 1-2, p. 6-27. 


\section{Résumé}

Une nouvelle approche critique est en train de s'implanter dans le champ de la connaissance. Pour les littéraires, une approche bioculturelle peut-elle contribuer à un renouveau épistémologique ? Le défi de cette nouvelle approche est de rassembler des savoirs constitués normalement séparément : il s'agit d'intégrer la biologie, l'anthropologie, la sociologie au vaste creuset des sciences humaines: c'est la «consilience». Cela suppose la reconnaissance préalable du rôle adaptatif que les narrations ont joué et jouent encore pour l'évolution d'Homo sapiens. Après un survol de la réception de Darwin dans les milieux non scientifiques, cet article propose un état présent de ce qu'il est convenu d'appeler l'approche bioculturelle ou biopoétique; il pose les grandes questions auxquelles cette discipline est confrontée, sans prétendre répondre à toutes, mais en espérant montrer tout l'intérêt que revêt ce nouveau terrain d'investigation. On trouvera en annexe une bibliographie de base propre à ce domaine.

\section{Abstract}

A new discipline is developing quite firmly as another field of cognition. Can literary Darwinism contribute to an epistemological renewal? The main challenge of this new approach may reside in integrating different domains that do not normally overlap: biology, anthropology, sociology, within the vast melting-pot of the humanities. This "Consilience" presupposes first to recognize the adaptive role that narrations have played in the evolution of Homo sapiens. After briefly reviewing Darwin's reception in nonscientific milieus, this article offers an update on what is now often called "Literary Darwinism", or biopoetics; it poses the great questions to which this discipline is confronted. Without claiming to offer an answer for all of them, it hopes at least to demonstrate the vital interest this new field of knowledge and thought may hold for the future. A basic bibliography with regard to this particular topic is annexed to this paper. 